\title{
Cardioprotective effects and underlying mechanism of Radix Salvia miltiorrhiza and Lignum Dalbergia odorifera in a pig chronic myocardial ischemia model
}

\author{
RUI LIN ${ }^{1,2^{*}}$, JIALIN DUAN $^{1 *}$, FEI MU ${ }^{1 *}$, HAIXU BIAN $^{1}$, MEINA ZHAO $^{1}$, MIN ZHOU $^{2}$, \\ YAO $\mathrm{LI}^{2}$, AIDONG WEN ${ }^{1}$, YONG YANG ${ }^{3}$ and MIAOMIAO XI ${ }^{1}$ \\ ${ }^{1}$ Department of Pharmacy, Xijing Hospital, Air Force Military Medical University, Xi'an, Shaanxi 710032; \\ ${ }^{2}$ School of Pharmacy, Shaanxi University of Chinese Medicine, Xianyang, Shaanxi 712046; ${ }^{3}$ Department of Pharmacy, \\ Sichuan Academy of Medical Sciences and Sichuan Provincial People's Hospital, \\ Chengdu, Sichuan 610072, P.R. China
}

Received January 25, 2018; Accepted August 9, 2018

DOI: $10.3892 / \mathrm{ijmm} .2018 .3844$

\begin{abstract}
Traditional Chinese medicines, including Radix Salvia miltiorrhiza (SM) and Lignum Dalbergia odorifera (DO) extracts, have historically been used to treat myocardial ischemia and other cardiovascular diseases. The volatile oil of DO (DOO) is one of the main components of DO. The aim of the present study was to assess the cardioprotective effects and possible underlying mechanisms of SM-DOO in pigs with ameroid constriction-induced chronic myocardial ischemia. An ameroid constrictor was placed around the left anterior descending coronary artery of pigs to induce chronic myocardial ischemia. At weeks 2, 6 and 8, myocardial injury markers and blood gas levels were detected. At week 8 , coronary angiography, echocardiography and hemodynamics analysis were performed to evaluate myocardial function. Following sacrifice, myocardial tissue was collected and subjected to morphological, histopathological and apoptosis assays. Western blotting was used to detect the protein expression of Bcl-2 associated X (Bax), Bcl-2, Akt, phosphorylated (p)-Akt, glycogen synthase kinase (GSK)-3 $\beta$ and p-GSK-3 $\beta$.
\end{abstract}

Correspondence to: Dr Miaomiao Xi, Department of Pharmacy, Xijing Hospital, Air Force Military Medical University, 15 Changle West Street, Xi'an, Shaanxi 710032, P.R. China

E-mail: miaomiaoxi2014@163.com

Dr Yong Yang, Department of Pharmacy, Sichuan Academy of Medical Sciences and Sichuan Provincial People's Hospital, 32 West Second Section First Ring Road, Chengdu, Sichuan 610072, P.R. China

E-mail: yyxpower@163.com

${ }^{*}$ Contributed equally

Key words: chronic myocardial ischemia, Radix Salvia miltiorrhiza, Lignum Dalbergia odorifera, cardioprotection, apoptosis, Akt, glycogen synthase kinase-3 $\beta$
It was revealed that SM-DOO treatment following chronic myocardial ischemia significantly downregulated the expression of myocardial injury markers, ameliorated myocardial oxygen consumption, increased collateralization, reduced regional cardiac dysfunction and limited the extent of myocardial damage. Furthermore, the results of an apoptosis assay revealed that the apoptosis rate was decreased, the expression of Bax decreased and Bcl-2 increased, and the ratio of $\mathrm{Bcl}-2 / \mathrm{Bax}$ was increased. Further experiments indicated that treatment with SM-DOO increased the phosphorylation of Akt and GSK-3 $\beta$. These findings suggest that SM-DOO treatment ameliorates myocardial injury in a chronic myocardial ischemia model, and that the underlying mechanisms responsible may be associated with the activation of the Akt/GSK-3 $\beta$ signal pathway. Thus, experimental evidence that SM-DOO may be an effective drug for the prevention and treatment of chronic myocardial ischemia in clinical applications has been provided.

\section{Introduction}

Myocardial ischemia, which is caused by an imbalance between the myocardial oxygen supply and myocardial oxygen requirement, can induce arrhythmia, cardiac dysfunction, myocardial infarction and sudden death (1). Although there are a number of novel drugs available for the treatment and prevention of myocardial ischemia, the mortality and morbidity continue to increase (2). It was previously reported that apoptosis may be an important step in the pathogenesis of myocardial injury, and exploring anti-apoptotic drugs may provide novel therapeutic opportunities for the treatment of myocardial ischemia (3).

Traditional Chinese medicine (TCM) has been used to treat cardiovascular diseases for more than 1,000 years with significant efficacy and minimal side effects (4). Among the herbs used in TCM, Radix Salvia miltiorrhiza (SM) and Lignum Dalbergia odorifera (DO) have been widely used in clinical and basic laboratory studies for the prevention and treatment of cardiovascular diseases, including in China (5-10). SM, known as Danshen in Chinese, has been used to treat cardiovascular 
diseases, including coronary heart disease, hyperlipidemia and cerebrovascular diseases (11). DO, known as Jiangxiang in Chinese, has been used to treat ischemia, necrosis, swelling, blood disorders and rheumatic pain (12). A number well-known Chinese medicines for treating cardiovascular disease contain SM and DO, including Guanxin II, Guanxin Danshen capsules, Xiangdan injection and Qishen Yiqi pills. The effect of the combination of SM-DO has been shown to be efficacious in clinical use $(13,14)$; however, there are few studies regarding their action and the underlying mechanisms responsible. In our previous experiment, the cardioprotective effects of SM and DO were evaluated in a rat myocardial ischemia-reperfusion model. The results indicated that combined treatment with SM and DO had significant cardioprotective effects, and that the combination of SM with DO volatile oil (DOO) (SM $5 \mathrm{~g} / \mathrm{kg} /$ day, DOO $0.5 \mathrm{ml} / \mathrm{kg} /$ day) was significantly more effective than SM, DO or DOO alone (15). However, the effects of SM-DOO in chronic myocardial ischemia have yet to be reported.

The aim of the present study was to investigate the cardioprotective effect and potential mechanisms of SM-DOO in a pig model of chronic myocardial ischemia. The major chemical components of SM and DOO were also determined using high-performance liquid chromatography (HPLC) and gas chromatography coupled with mass spectrometry (GC-MS), respectively.

\section{Materials and methods}

Materials and reagents. SM extract was purchased from Xi'an Honson Biotechnology Co., Ltd. (Xi'an, China; batch no. 161025). The major components were hydrophilic salvanolic acid and hydrophobic tanshinones, as evaluated by HPLC analysis. DOO was purchased from Jishui Jinhai Natural Perfume Oil Technology Co., Ltd. (Jiangxi, China; batch no. XC20160918) and the major components were evaluated by GC-MS. Standards for danshensu, rosmarinic acid, salvianolic acid $\mathrm{B}$, salvianolic acid $\mathrm{A}$, protocatechuic acid, protocatechuic aldehyde, dihydrotanshinone I, cryptotanshinone, tanshinone $\mathrm{II}_{\mathrm{A}}$ and tanshinon $\mathrm{II}_{\mathrm{A}}$ silate sodium were purchased from Shanghai ANPEL Laboratory Technologies, Inc. (Shanghai, China; batch no. Q2880010). Acetonitrile was purchased from Thermo Fisher Scientific, Inc. (Waltham, MA, USA). All other reagents were analysis grade.

Deionized water was prepared using a Milli-Q water purification system (EMD Millipore, Bedford, MA, USA). 2,3,5-triphenyltetrazolium chloride (TTC) was purchased from Sigma-Aldrich (Merck KGaA, Darmstadt, Germany). Pig creatine kinase-MB (CK-MB), cardiac troponin I (cTnI) and myoglobin (Myo) ELISA kits were bought from Xitang Biology Technology Company (Shanghai, China). Lactate dehydrogenase (LDH), alanine aminotransferase (ALT) and aspartate aminotransferase (AST) test kits were purchased from Nanjing Jiancheng Bioengineering Institute (Nanjing, China). All primary antibodies, including Bcl-2 associated $\mathrm{X}$ (Bax; cat. no. 2772), Bcl-2 (cat. no. 2870), Akt (cat. no. 9272), phosphorylated (p)-Akt (cat. no. 9271), glycogen synthase

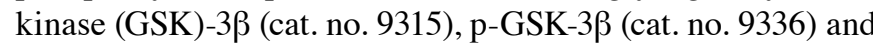
$\beta$-actin (cat. no. 4970), were purchased from Cell Signaling Technologies, Inc. (Danvers, MA, USA). The secondary antibody (anti-rabbit IgG-B; cat. no. sc-53804) was purchased from Santa Cruz Biotechnology, Inc. (Dallas, TX, USA).

Herbal extraction. SM was dried at $50^{\circ} \mathrm{C}$ and ground into powder $(<1 \mathrm{~mm})$. SM (150 g) was soaked in 8-fold the volume of water for $30 \mathrm{~min}$ at room temperature and extracted for $3 \mathrm{~h}$ $(1.5 \mathrm{~h} /$ reflux $)$, followed by reflux extraction with $75 \%$ ethanol. The filtrates were combined, concentrated under reduced pressure and freeze-dried. DOO was isolated by steam distillation for $5 \mathrm{~h}$, with a yield of $0.5 \% \mathrm{DOO}$, which was stored at $4^{\circ} \mathrm{C}$.

Preparation of standard solutions. Primary standard solutions of the 10 components were prepared by dissolving the solid standards in methanol. Working mixed standard solutions (40 $\mu \mathrm{g} / \mathrm{ml}$ ) were prepared by mixing and diluting the stock solution with methanol. The mixed standard solution was filtered through a $0.22-\mu \mathrm{m}$ membrane before HPLC analysis.

Preparation of herb solutions. SM was dried at a temperature of $50^{\circ} \mathrm{C}$ and ground into powder. Subsequently, $0.25 \mathrm{~g}$ $\mathrm{SM}$ was soaked in 8-fold the volume of water for $30 \mathrm{~min}$ at room temperature and extracted for $4 \mathrm{~h}$, followed by ultrasound-assisted extraction for 30 min with $80 \%$ methanol. The suspension was filtered, concentrated and diluted to a $50-\mathrm{ml}$ volume with methanol. The solution was filtered through a $0.22-\mu \mathrm{m}$ membrane prior to HPLC analysis.

An aliquot of $200 \mu \mathrm{l}$ DOO was diluted in $20 \mathrm{ml}$ of ethyl acetate and dried over anhydrous sodium sulfate. The supernatant was filtered through a $0.22-\mu \mathrm{m}$ membrane prior to GC-MS analysis.

Chromatographic conditions. HPLC analysis was performed using an Agilent 1260 HPLC system (Agilent Technologies, Santa Clara, CA, USA) and chromatographic separation was accomplished using an Agilent TC-C18 column (4.6x250 mm, $5 \mu \mathrm{m})$. The column temperature was set at $30^{\circ} \mathrm{C}$ with a $20-\mu 1$ injection volume. The mobile phase consisted of $0.2 \%$ formic acid (v/v) in water (A) and acetonitrile (B). The gradient program used was as follows: $10 \% \mathrm{~B}$ to $30 \% \mathrm{~B}$ (0-15 min), $30 \% \mathrm{~B}$ to $32 \% \mathrm{~B}(15-40 \mathrm{~min}), 32 \% \mathrm{~B}$ to $55 \% \mathrm{~B}(40-55 \mathrm{~min})$, $55 \% \mathrm{~B}$ to $67 \% \mathrm{~B}(55-65 \mathrm{~min}), 67 \% \mathrm{~B}$ to $72 \% \mathrm{~B}(65-75 \mathrm{~min})$, $72 \% \mathrm{~B}$ to $85 \% \mathrm{~B}(75-85 \mathrm{~min})$ and $100 \% \mathrm{~B}(85-100 \mathrm{~min})$. The flow rate was set at $0.8 \mathrm{ml} / \mathrm{min}$ throughout the analysis, and UV detection was set at $270 \mathrm{~nm}$.

The components of DOO were analyzed using an Agilent 7890A gas chromatograph coupled with an Agilent 5975C mass selective detector (Agilent Technologies). Chromatographic separation was performed using an HP-5MS capillary column $(30 \mathrm{~m} \times 0.25 \mathrm{~mm} \times 0.25 \mu \mathrm{m}$ film thickness, Agilent Technologies). The split ratio was 15:1, and the injection volume was set to $2 \mu \mathrm{l}$. The inlet and ion source temperatures were set to $260^{\circ} \mathrm{C}$ and $230^{\circ} \mathrm{C}$, respectively. The helium flow rate was set to $1.0 \mathrm{ml} / \mathrm{min}$. The oven temperature was initially $60^{\circ} \mathrm{C}$, followed by a $1.5^{\circ} \mathrm{C} / \mathrm{min}$ increase to $105^{\circ} \mathrm{C}$ for $10 \mathrm{~min}$, then a $1^{\circ} \mathrm{C} / \mathrm{min}$ increase to $115^{\circ} \mathrm{C}$ for $10 \mathrm{~min}$, a $2.5^{\circ} \mathrm{C} / \mathrm{min}$ increase to $160^{\circ} \mathrm{C}$ for $5 \mathrm{~min}$ and finally to $260^{\circ} \mathrm{C}$ at a heating rate of $20^{\circ} \mathrm{C} / \mathrm{min}$. MS was used in full scan mode with the acquisition range set to $30-800 \mathrm{~m} / \mathrm{z}$. GC-MS post-run analysis was performed, and the main components were identified using the library of the National Institute of Standards and 
Technology (NIST) (16). The peak area normalization method was used to establish the relative content of each substance in the sample.

Animals and experimental protocol. A total of 18 male Yorkshire pigs $(25-30 \mathrm{~kg})$ were purchased from the Institute of Experiment Animals at the Fuwai Hospital Experimental Animal Center (Beijing, China) and kept in an animal room at $25 \pm 1^{\circ} \mathrm{C}$ with $55 \pm 5 \%$ humidity. The Animal Welfare and Ethics Committee of Fuwai Hospital, Chinese Academy of Medical Sciences approved all experiments [approval no. 0072-1-27-HX (F)]. After one week of acclimation, the pigs were randomized into 3 groups as follows: i) Sham group $(n=6)$, oral administration of regular diet and sham-operated without ameroid constrictor implantation; ii) Model group $(n=6)$, oral administration of regular diet; iii) SM-DOO group $(\mathrm{n}=6)$, oral administration of regular diet with $1 \mathrm{~g} / \mathrm{kg} /$ day SM and $0.1 \mathrm{ml} / \mathrm{kg} / \mathrm{day}$ DOO. The dosages of SM and DOO were determined based on our previous studies, and adjusted for pigs according to a previously published method $(15,17)$. Pigs were provided free access to water and were fed twice a day. All animals were observed during feeding to ensure the entire portion of food was consumed.

At 4 weeks after the dietary modification, pentobarbital sodium $(25 \mathrm{mg} / \mathrm{kg})$ was administered intramuscularly (i.m.) to induce anesthesia. Animals were intubated, ventilated with oxygen at $1.5-2 \mathrm{l} / \mathrm{min}$ and anesthetized with $3 \%$ isoflurane. The pigs were right lateral decubitus, and a small thoracotomy was performed in intercostal segments 3 and 4 by sterile techniques. An ameroid constrictor (Research Instruments NW, Inc., Lebanon, OR, USA) with a 2.5-mm internal diameter was implanted around the left anterior descending artery (LAD). The ameroid constrictor was designed to achieve myocardial ischemia by inducing progressive coronary artery stenosis with a progression similar to that observed in humans. The pericardium was closed using a 6-0 polypropylene suture (Ethicon, Inc., Somerville, NJ, USA) and the chest was sutured closed. After the surgery, penicillin $(6,400,000 \mathrm{U} /$ day, i.m.) was administered to all pigs to prevent infection.

After 8 weeks, echocardiography, X-ray coronary angiography and hemodynamics analysis were performed. All surgical protocols were designed to minimize the suffering of the pigs. A schematic presentation of the study protocol is shown in Fig. 1.

Measurement of myocardial injury markers. Blood samples were collected in heparinized tubes, allowed to clot for $30 \mathrm{~min}$ and centrifuged at $2,285 \mathrm{x} \mathrm{g}$ at $4^{\circ} \mathrm{C}$ for $15 \mathrm{~min}$. Myocardial injury markers, including CK-MB, LDH, ALT, AST, cTnI and Myo, were measured using assay kits or pig-specific ELISA kits according to the manufacturers' protocols.

Blood gas analysis. At weeks 2, 6 and 8, pH, $\mathrm{PO}_{2}$ and $\mathrm{PCO}_{2}$ were measured in arterial blood samples taken from the femoral artery (500-700 $\mu \mathrm{l})$ using pre-heparinized syringes $(1,000 \mathrm{IU} / \mathrm{ml})$ and analyzed using an i-STAT 1 blood gas system (Abbott Laboratories, Chicago, IL, USA).

Echocardiography. Echocardiography was performed at the end of the study using a Vivid 9 machine (GE Vingmed
Ultrasound AS, Horten, Norway). Left ventricular end-diastolic diameter (LVEDd), left ventricular end-systolic diameter (LVEDs), end-diastolic volume (EDV), end-systolic volume (ESV), ejection fraction (EF) and fractional shortening (FS) were recorded to assess cardiac function. The FS value was calculated based on the following equation: FS $(\%)=[($ LVEDd-LVEDs $) / L V E D d] \times 100 \%$. Images were stored and analyzed using Echopac software (GE Vingmed Ultrasound AS).

X-ray coronary angiography. X-ray coronary angiography (GE Medical Systems, Milwaukee, WI, USA) with iopromide (Juste SAQF, Madrid, Spain) was performed on all experimental animals to verify the occlusion of the LAD and assess collateral vessel filling at the end of the experiment. The grade of collateral filling was scored using a standard 4-point scale as follows (18): $0=$ no collateral filling; $1=$ minimal collateral filling; $2=$ moderate collateral filling, but less than the contralateral or ipsilateral non-infarct coronary artery; $3=$ normal collateral filling compared with the contralateral or ipsilateral non-infarct coronary artery.

Hemodynamic monitoring. The heart rate (HR), mean arterial blood pressure (MAP), left ventricular systolic pressure (LVSP), left ventricular end-diastolic pressure (LVEDP), left ventricular maximum upstroke velocity $\left(+\mathrm{dp} / \mathrm{dt}_{\max }\right)$ and left ventricular maximum descent velocity $\left(-\mathrm{dp} / \mathrm{dt}_{\max }\right)$ were recorded using a pressure transducer (Millar Instruments, Houston, TX, USA) connected to an anesthesia monitor (Philips, Eindhoven, Netherlands).

Morphometric and histopathological examination. Animals were sacrificed by intravenous injection with potassium chloride after the imaging study, and their hearts were explanted. Hearts were serially sliced and incubated with $2 \%$ TTC for $20 \mathrm{~min}$ at $37^{\circ} \mathrm{C}$. An Image-Pro Plus image system (Media Cybernetics, Bethesda, MD, USA) was used to analyze the infarct (white) and non-infarct (red) areas. The percentage of myocardial infarct was calculated as infarct area divided by total area.

The left ventricle was harvested, fixed in $4 \%$ paraformaldehyde, embedded in paraffin and sectioned. Sections were stained with hematoxylin and eosin (H\&E) for histological examination. The pathology of heart sections was investigated using a light microscope, and photomicrographs were captured.

Measurement of myocardial apoptosis. Myocardial apoptosis was measured using an In-Situ Cell Death Detection kit (Wanlei Bio, Shenyang, China) according to the manufacturer's protocol. Myocardial slices were subjected to terminal deoxynucleotidyl transferase-mediated dUTP nick end-labeling (TUNEL) staining combined with a DAPI (Sigma-Aldrich; Merck KGaA) counterstain. Images were obtained under a fluorescence microscope at x400 magnification (Nikon Corporation, Tokyo, Japan), to confirm the successful labeling of apoptotic cells and nuclei. The percentage of TUNEL-positive cells was calculated as the number of apoptotic cells divided by the total number of nuclei x 100. 


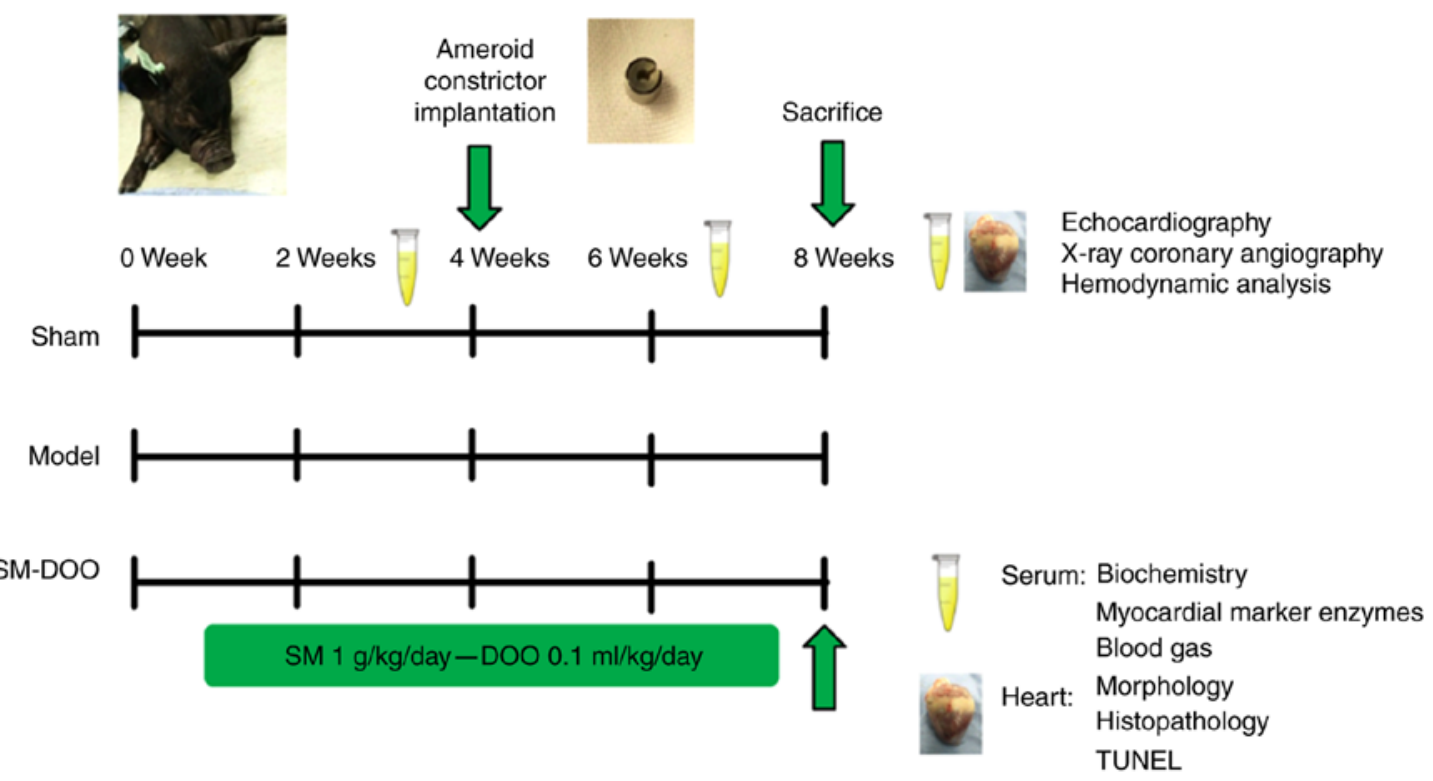

Figure 1. Schematic presentation of the study protocol. Pigs were randomly divided into 3 groups: Sham, Model and SM-DOO (n=6). The SM-DOO group received SM $(1 \mathrm{~g} / \mathrm{kg} / \mathrm{day})$ and DOO $(0.1 \mathrm{ml} / \mathrm{kg} /$ day $)$ orally for 8 weeks. At 4 weeks, an ameroid constrictor was placed around the left anterior descending coronary artery in the Model and SM-DOO groups. At weeks 2, 4 and 8, biochemical markers, myocardial marker enzymes and blood gas constituents were measured in the serum. After 8 weeks, echocardiography, X-ray coronary angiography and hemodynamic analysis were performed. Myocardial tissue underwent morphological, histopathological, apoptosis and western blot analysis to evaluate the cardioprotective effects and underlying mechanisms of SM-DOO. SM, Radix Salvia miltiorrhiza; DOO, Lignum Dalbergia odorifera volatile oil; TUNEL, terminal deoxynucleotidyl transferase-mediated dUTP nick end-labeling.

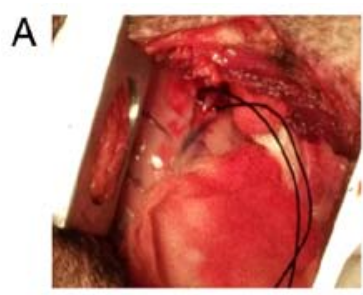

Sham

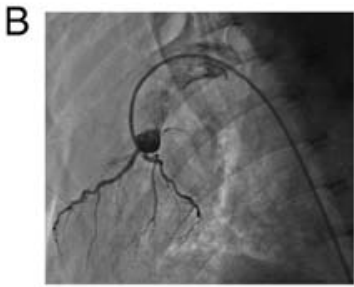

Sham

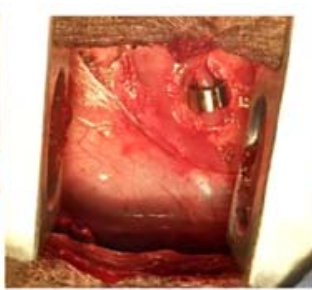

Chronic myocardial ischemia

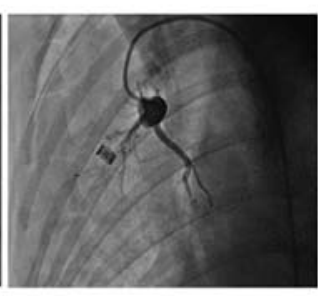

Model

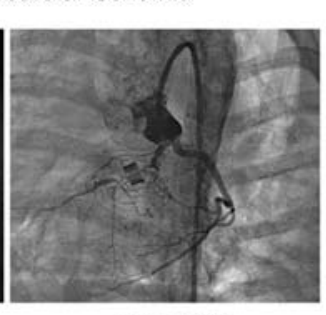

SM-DOO

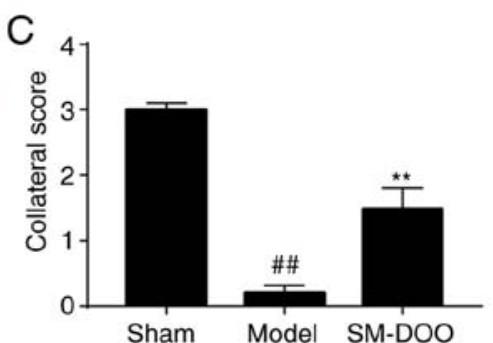

Sham Model SM-DOO

Figure 2. At 4 weeks after ameroid constrictor implantation, coronary angiography was performed. (A) The Sham group underwent the surgical procedure without ameroid constrictor implantation, whereas in the Model and SM-DOO groups a 2.5-mm ameroid constrictor was implanted around the LAD to produce chronic myocardial ischemia. (B) Representative coronary angiographies from each group. No coronary stenosis was observed in the Sham group. No collateral vessel filling was observed in the Model group. In the SM-DOO group, collateral vessel filling was observed with distal filling of the LAD. (C) Assessment of the myocardial collateral grade revealed increased collateral vessel filling in the SM-DOO group. All values are presented as the mean \pm standard deviation. ${ }^{\# \#} \mathrm{P}<0.01$ vs. Sham group; ${ }^{* *} \mathrm{P}<0.01$ vs. Model group. SM, Radix Salvia miltiorrhiza; DOO, Lignum Dalbergia odorifera volatile oil; LAD, left anterior descending artery.

Western blotting analysis. Myocardial tissues were homogenized in RIPA buffer containing $1 \%$ PMSF and $1 \%$ protease inhibitor cocktail (Nanjing Jiancheng Bioengineering Institute), and the supernatant was harvested by concentration $\left(18,447 \mathrm{x}\right.$ g at $4^{\circ} \mathrm{C}$ for $\left.10 \mathrm{~min}\right)$. The protein concentration was determined based on the BCA method using a Protein Quantitative Analysis kit (Nanjing Jiancheng Bioengineering Institute). The lysates were separated by $10 \%$ SDS-PAGE.
Following separation, the proteins were transferred onto polyvinyl difluoride membranes, blocked for $1 \mathrm{~h}$ at $37^{\circ} \mathrm{C}$ with 5\% non-fat dried milk, washed 3 times with Tween in Tris-buffered saline, and incubated with primary antibodies

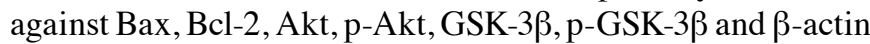
(dilution, $1: 1,000$ ) overnight at $4^{\circ} \mathrm{C}$. Subsequent to washing 3 times as described above, the membranes were incubated with secondary antibodies (dilution, 1:5,000) at $37^{\circ} \mathrm{C}$ for $1 \mathrm{~h}$. 

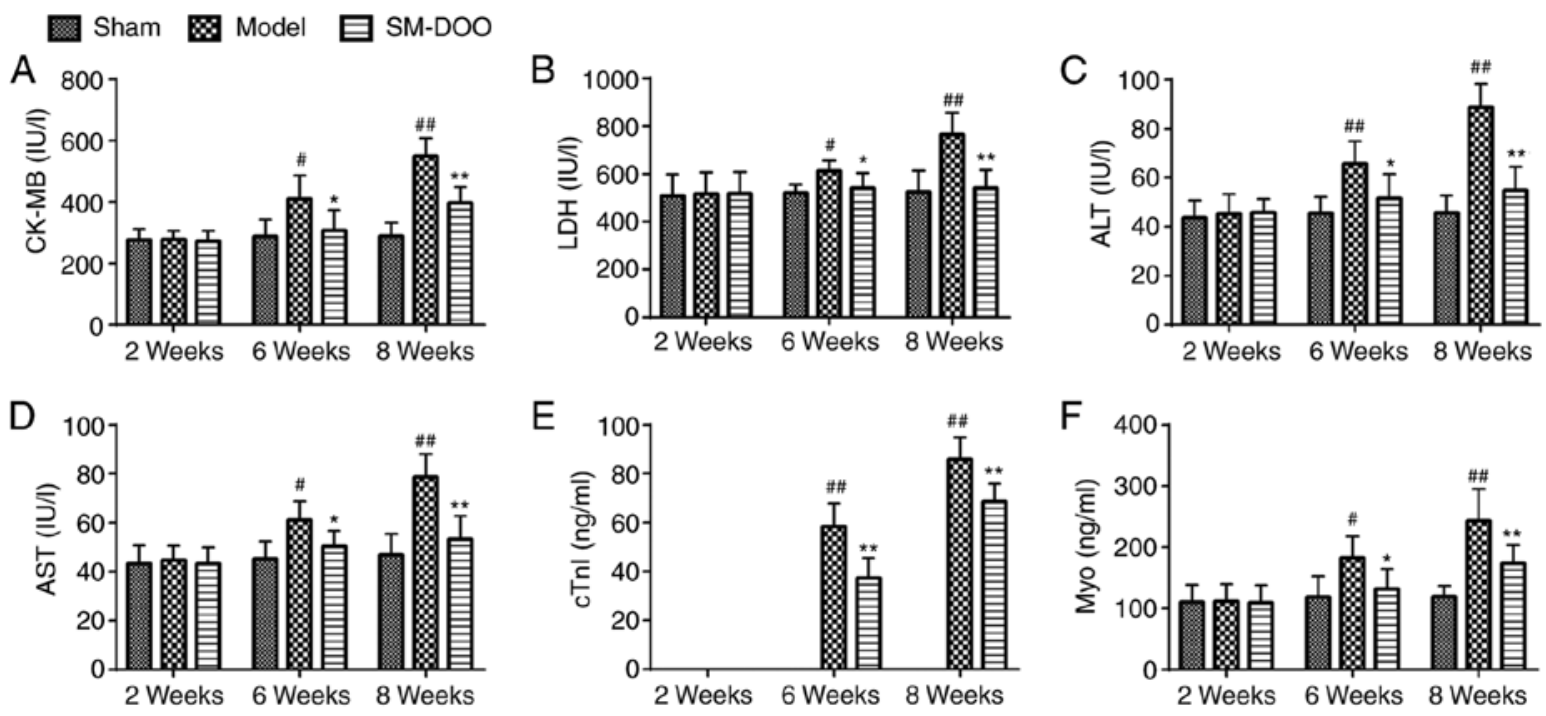

Figure 3. Effects of SM-DOO on myocardial injury markers at weeks 2, 6 and 8 in pigs with chronic myocardial ischemia, including (A) CK-MB, (B) LDH, (C) ALT, (D) AST, (E) cTnI and (F) Myo. All values are presented as the mean \pm standard deviation. ${ }^{\#} \mathrm{P}<0.05$ and ${ }^{\# \#} \mathrm{P}<0.01$ vs. Sham group; ${ }^{*} \mathrm{P}<0.05$ and ${ }^{* *} \mathrm{P}<0.01$ vs. Model group. SM, Radix Salvia miltiorrhiza; DOO, Lignum Dalbergiae odoriferae volatile oil; CK-MB, Pig creatine kinase-MB; LDH, lactate dehydrogenase; ALT, alanine aminotransferase; AST, aspartate aminotransferase; cTnI, cardiac troponin I; Myo, myoglobin.
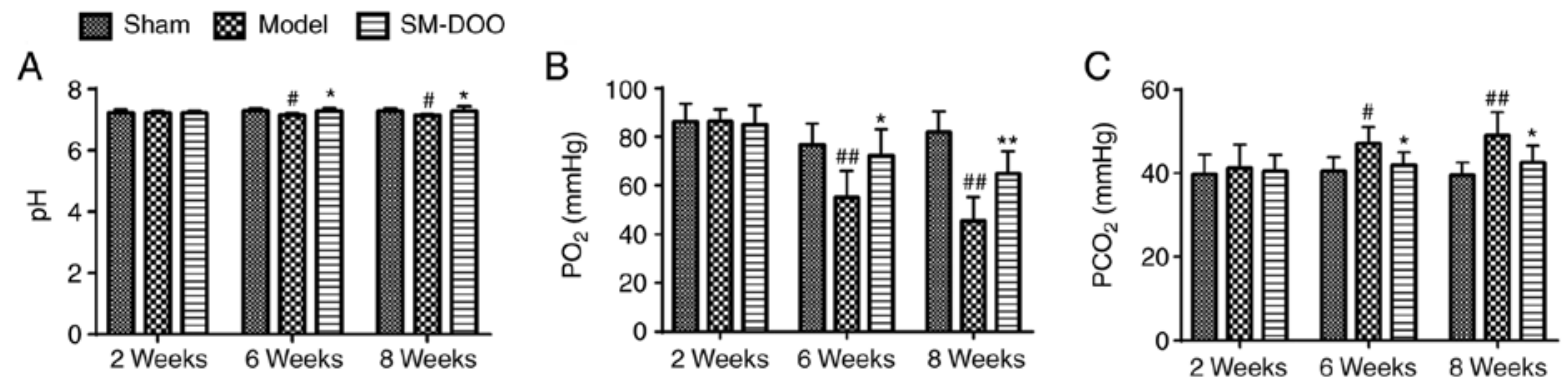

Figure 4. Effects of SM-DOO on myocardial oxygen consumption. At weeks 2, 6 and 8 the (A) pH, (B) $\mathrm{PO}_{2}$ and (C) $\mathrm{PCO}_{2}$ levels in the blood were measured. All values are presented as the mean \pm standard deviation. ${ }^{\#} \mathrm{P}<0.05$ and ${ }^{\# \#} \mathrm{P}<0.01$ vs. Sham group; ${ }^{*} \mathrm{P}<0.05$ and ${ }^{* *} \mathrm{P}<0.01$ vs. Model group. SM, Radix Salvia miltiorrhiza; DOO, Lignum Dalbergia odorifera volatile oil.

Immunoreactive bands were detected using the enhanced chemiluminescence method and semi-quantified using Image J Software version 1.47 (National Institutes of Health, Bethesda, MD, USA).

Statistical analysis. Statistical analysis was conducted using GraphPad Prism version 6.02 (GraphPad Software, Inc., La Jolla, CA, USA). All data are presented as the mean \pm standard deviation. One-way analysis of variance with Tukey's multiple comparison post-hoc test was performed to analyze the data. $\mathrm{P}<0.05$ was considered to represent a statistically significant difference.

\section{Results}

Evaluation of the chronic myocardial ischemia model and effect of SM-DOO on collateral vessel filling. The ameroid constrictor was used to induce coronary closure. Coronary angiography indicated that the rate of coronary stenosis reached $98-100 \% 4$ weeks after ameroid constrictor implantation, indicating that chronic myocardial ischemia was stably established. As shown in Fig. 2, coronary angiography revealed
LAD stenosis with no evidence of collateral vessel filling in the Model group ( $0.20 \pm 0.11)$; however, collateral filling was observed in the SM-DOO group (1.49 \pm 0.31$)$. Collateral scores were significantly increased by the administration of SM-DOO compared with the Model group $(\mathrm{P}<0.01)$.

Effect of SM-DOO on myocardial injury markers. As presented in Fig. 3, no differences were observed in CK-MB, LDH, ALT, AST, cTnI and Myo between the 3 groups at week 2. CK-MB, LDH, ALT, AST, cTnI and Myo levels in the Model group were higher compared with the Sham group at weeks 6 and 8. In the Model group, at week 8, the expression of myocardial injury markers was higher compared with at week 6. Conversely, CK-MB, LDH, ALT, AST, cTnI and Myo were significantly decreased in the SM-DOO group compared with the Model group $(\mathrm{P}<0.05$ or $\mathrm{P}<0.01)$, demonstrating that SM-DOO ameliorated injury in chronic myocardial ischemia.

Effect of SM-DOO on myocardial oxygen consumption. To investigate whether the cardioprotective effects of SM-DOO were mediated by affecting myocardial oxygen consumption, blood gas was quantified. As presented in Fig. 4, pH, $\mathrm{PO}_{2}$ 
Table I. Movement of the left ventricular anterior wall at the mitral level, papillary muscle level and apical level.

\begin{tabular}{|c|c|c|c|c|c|c|}
\hline \multirow[b]{2}{*}{ Group } & \multicolumn{2}{|c|}{ Mitral level } & \multicolumn{2}{|c|}{ Papillary muscle level } & \multicolumn{2}{|c|}{ Apical level } \\
\hline & End-systolic & End-diastolic & End-systolic & End-diastolic & End-systolic & End-diastolic \\
\hline Sham & $1.067 \pm 0.121$ & $0.773 \pm 0.103$ & $1.100 \pm 0.141$ & $0.750 \pm 0.084$ & $1.100 \pm 0.126$ & $0.700 \pm 0.063$ \\
\hline Model & $0.800 \pm 0.063^{\mathrm{b}}$ & $0.550 \pm 0.055^{\mathrm{b}}$ & $0.850 \pm 0.138^{a}$ & $0.583 \pm 0.075^{b}$ & $0.767 \pm 0.103^{b}$ & $0.533 \pm 0.052^{\mathrm{b}}$ \\
\hline SM-DOO & $0.950 \pm 0.105^{\mathrm{c}}$ & $0.683 \pm 0.075^{\mathrm{c}}$ & $1.067 \pm 0.105^{\mathrm{c}}$ & $0.717 \pm 0.075^{c}$ & $0.950 \pm 0.085^{\mathrm{c}}$ & $0.667 \pm 0.103^{c}$ \\
\hline
\end{tabular}

All values are presented as the mean \pm standard deviation. ${ }^{\mathrm{a}} \mathrm{P}<0.05,{ }^{\mathrm{b}} \mathrm{P}<0.01$ vs. Sham group, ${ }^{\mathrm{c}} \mathrm{P}<0.05$ vs. Model group. SM, Radix Salvia miltiorrhiza; DOO, Lignum Dalbergia odorifera volatile oil.
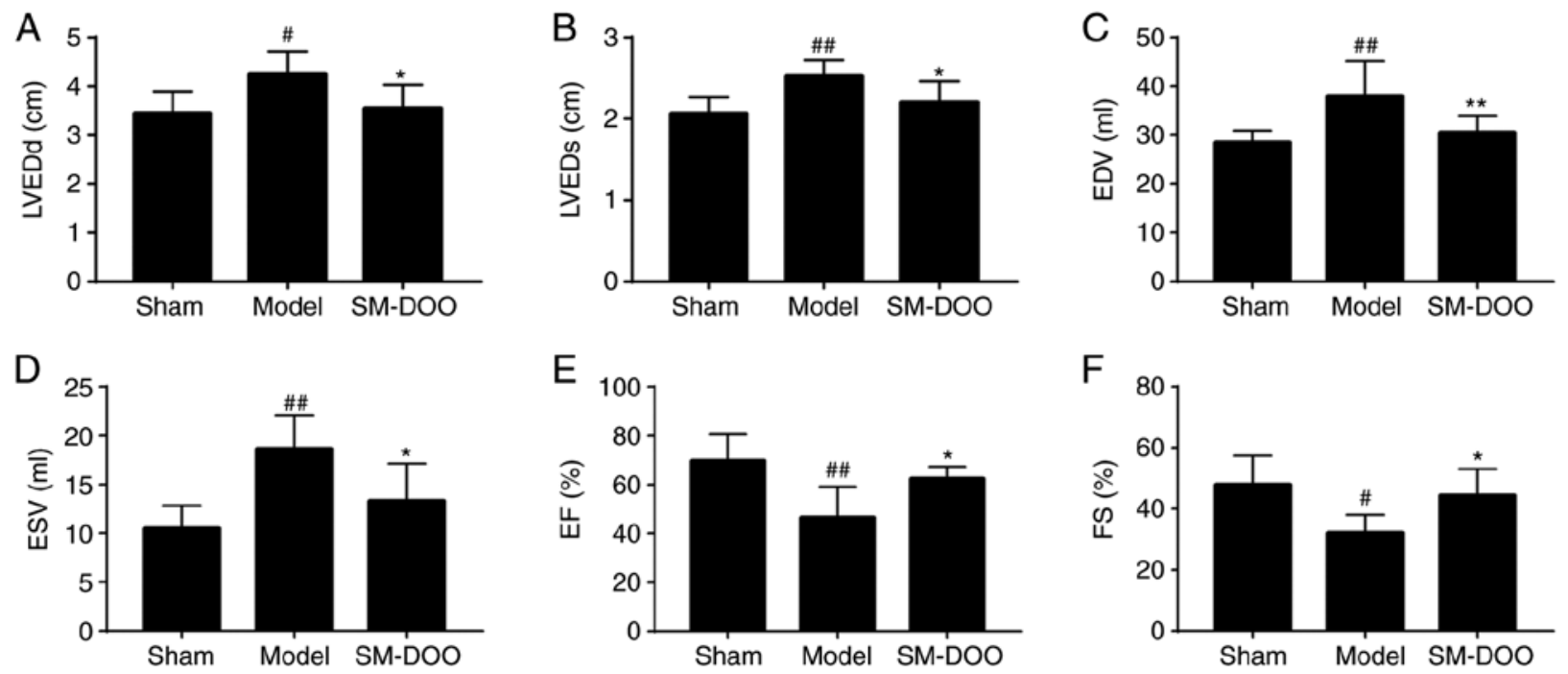

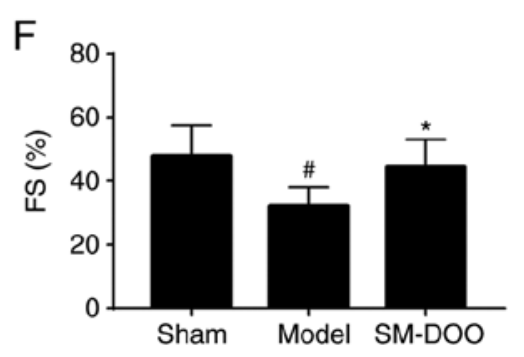

Figure 5. Effects of SM-DOO on left ventricular function after chronic myocardial ischemia, including (A) LVEDd, (B) LVEDs, (C) EDV, (D) ESV, (E) EF and (F) FS. All values are presented as the mean \pm standard deviation. ${ }^{\#} \mathrm{P}<0.05$ and ${ }^{\# \#} \mathrm{P}<0.01$ vs. Sham group; ${ }^{*} \mathrm{P}<0.05$ and ${ }^{* *} \mathrm{P}<0.01$ vs. Model group. LVEDd, left ventricular end-diastolic diameter; LVEDs, left ventricular end-systolic diameter; EDV, end-diastolic volume; ESV, end-systolic volume; EF, ejection fraction; FS, fractional shortening.

and $\mathrm{PCO}_{2}$ were the same in all 3 groups prior to the induction of chronic myocardial ischemia. By weeks 6 and 8, the arterial $\mathrm{pH}$ and $\mathrm{PO}_{2}$ were markedly lower, while the $\mathrm{PCO}_{2}$ was higher in the Model group than that in the Sham group $(47.13 \pm 3.91$ vs. $40.57 \pm 3.29 \mathrm{mmHg}$ at week $6 ; 49.05 \pm 5.56 \mathrm{vs}$ $39.46 \pm 3.10 \mathrm{mmHg}$ at week 8). Thus, compared with the Model group, $\mathrm{SM}-\mathrm{DOO}$ markedly ameliorated the effects on $\mathrm{pH}, \mathrm{PO}_{2}$ and $\mathrm{PCO}_{2}$ induced by myocardial ischemia.

Effects of SM-DOO on left ventricular function. To assess the cardioprotective effects of SM-DOO, left ventricular function was examined. Compared with the Sham group, the mitral and apical levels in the Model group were reduced, the papillary muscle was thinned, and the left ventricular anterior wall moments were weakened (Table I). Additionally, in the Model group, the echocardiography parameters of LVEDd, LVEDs, EDV and ESV were significantly increased, while EF and FS were markedly reduced $(\mathrm{P}<0.01$ or $\mathrm{P}<0.05)$. The results indicated that the left ventricular function in the Model group was markedly altered. Conversely, the effects on the left ventricular anterior wall thickness and moments were ameliorated in the SM-DOO group; the levels of LVEDd, LVEDs, EDV and ESV were significantly reduced, whereas EF and FS were increased following treatment with SM-DOO $(62.5 \pm 4.848 \%$ for $\mathrm{EF}$, $\mathrm{P}<0.05 ; 44.5 \pm 8.526 \%$ for FS; $\mathrm{P}<0.01$; Fig. 5 ).

Effects of SM-DOO on hemodynamic factors. As shown in Fig. 6, the hemodynamic parameters indicated that the left ventricular function of the Model group was significantly decreased compared with the Sham group. In the SM-DOO group, HR, LVEDP and $-\mathrm{dp} / \mathrm{dt}_{\max }$ were markedly reduced $(\mathrm{P}<0.05$ or 0.01$)$, whereas MAP, LVSP and $+\mathrm{dp} / \mathrm{dt}_{\max }$ were significantly increased, compared with the Model group $(102.176 \pm 22.203$ vs. $67.079 \pm 16.378 \mathrm{mmHg}$ for MAP; $115.522 \pm 21.528$ vs. $83.206 \pm 9.189 \mathrm{mmHg}$ for LVSP; and $2,017.917 \pm 548.228$ vs. $1,244.587 \pm 284.314 \mathrm{mmHg} / \mathrm{sec}$ for $\left.+\mathrm{dp} / \mathrm{dt}_{\max } ; \mathrm{P}<0.05\right)$.

Effects of SM-DOO on myocardial damage. After the pigs were sacrificed, hearts were collected for morphometric and histopathological analysis. Cardiac sections were stained with TTC to compare the infarct size between the 3 groups. As shown in Fig. 7A, the mean infarct size in the Model group was larger than that in the SM-DOO group. The infarct size was 

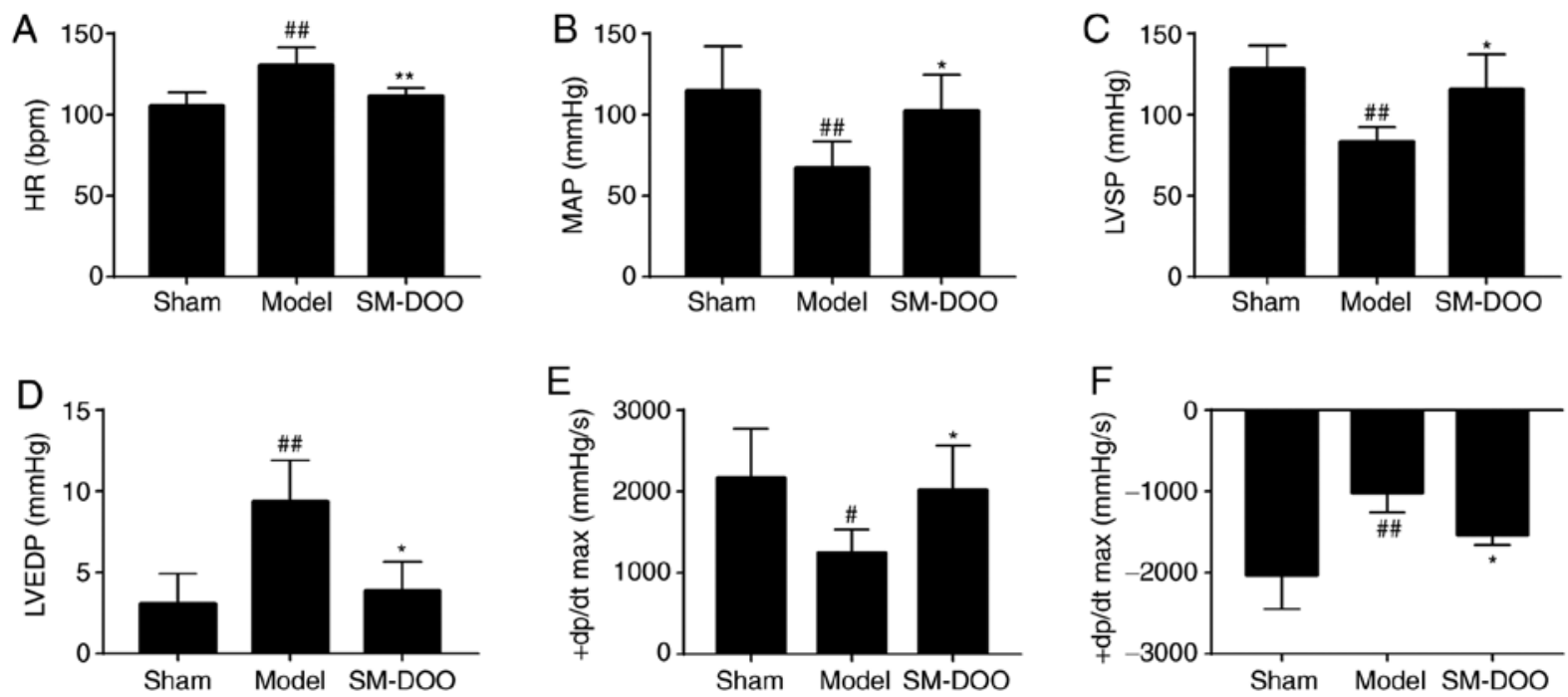

Figure 6. Effects of SM-DOO on hemodynamics. The (A) HR, (B) MAP, (C) LVSP, (D) LVEDP, (E) $+\mathrm{dp} / \mathrm{dt}_{\max }$ and (F) -dp/dt max $_{\text {ax }}$ levels in each group were determined. All values are presented as the mean $\pm \mathrm{SD} .{ }^{*} \mathrm{P}<0.05$ and ${ }^{\# \#} \mathrm{P}<0.01$ vs. Sham group, ${ }^{*} \mathrm{P}<0.05$ and ${ }^{* * *} \mathrm{P}<0.01$ vs. Model group. SM, Radix Salvia miltiorrhiza; DOO, Lignum Dalbergia odorifera volatile oil; HR, heart rate; MAP, mean arterial blood pressure; LVSP, left ventricular systolic pressure; LVEDP, left ventricular end-diastolic pressure; $+\mathrm{dp}_{\mathrm{dt}} \mathrm{d}_{\max }$, left ventricular maximum upstroke velocity; - $\mathrm{dp} / \mathrm{dt}_{\max }$, left ventricular maximum descent velocity.

A

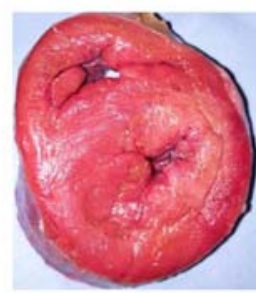

Sham

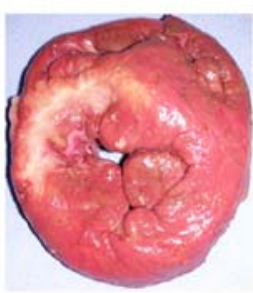

Model

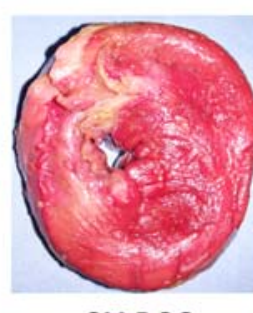

SM-DOO

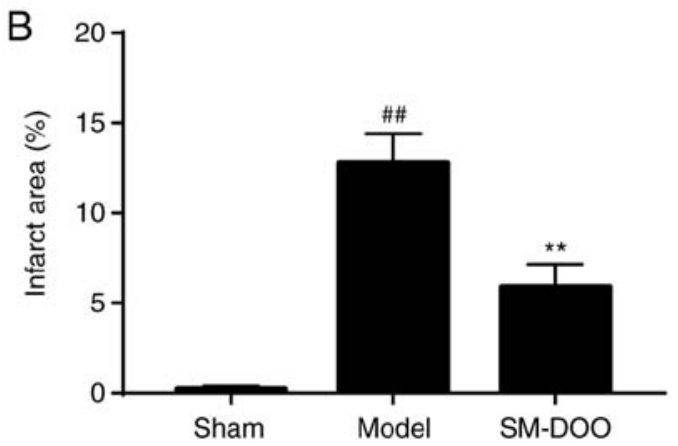

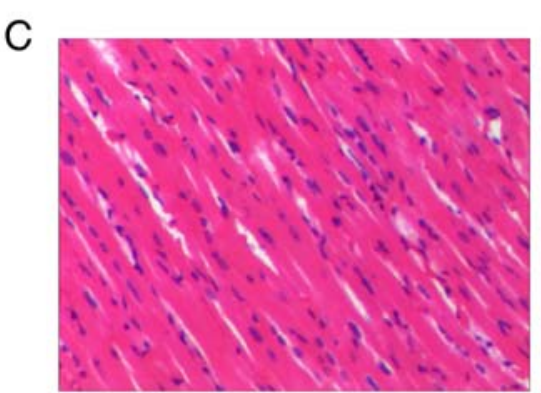

Sham

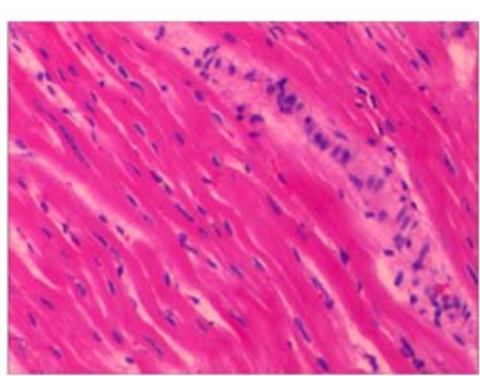

Model

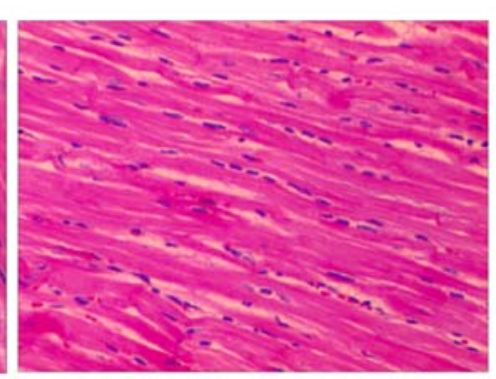

SM-DOO

Figure 7. Effects of SM-DOO on myocardial infarct size after myocardial injury induced by ameroid constrictor implantation. (A) The infarct area was stained white, while the non-infarct area was stained red. (B) Infarct area (\%) relative to the total area. (C) Representative photomicrographs of left ventricles of the pigs with hematoxylin and eosin staining (magnification, $\mathrm{x} 200$ ) following chronic myocardial ischemia. All values are presented as the mean \pm standard deviation. ${ }^{\# \#} \mathrm{P}<0.01$ vs. Sham group; ${ }^{* *} \mathrm{P}<0.01$ vs. Model group. SM, Radix Salvia miltiorrhiza; DOO, Lignum Dalbergia odorifera volatile oil.

significantly decreased in the SM-DOO group $(5.91 \pm 1.25 \%)$ compared with the Model group $(12.93 \pm 1.68 \%$; $\mathrm{P}<0.01$; Fig. 7B). Thus, SM-DOO treatment reduced the myocardial infarct size after chronic myocardial ischemia.

As presented in Fig. 7C, the myocardium was H\&E stained for histological examination. Myocardial tissues in the Sham group exhibited no abnormalities. As expected, chronic myocardial ischemia induced evident injury to the myocardial structure, including chronic inflammatory cells, edema in the myocardial fibers and ruptured myocardial fibers in the Model group. Myocardial alterations following chronic myocardial ischemia, in particular, disruption to the myocardial fibers, were significantly ameliorated by SM-DOO.

Effects of SM-DOO on myocardial apoptosis and Bax and Bcl-2 expression. As shown in Fig. 8A, more apoptotic cells were observed in the Model group compared with the Sham group. The percentage of TUNEL-positive cells was significantly lower in the SM-DOO group $(19.78 \pm 2.51 \%)$ compared with the Model group (28.20 $\pm 5.33 \%)$, as shown in 
A
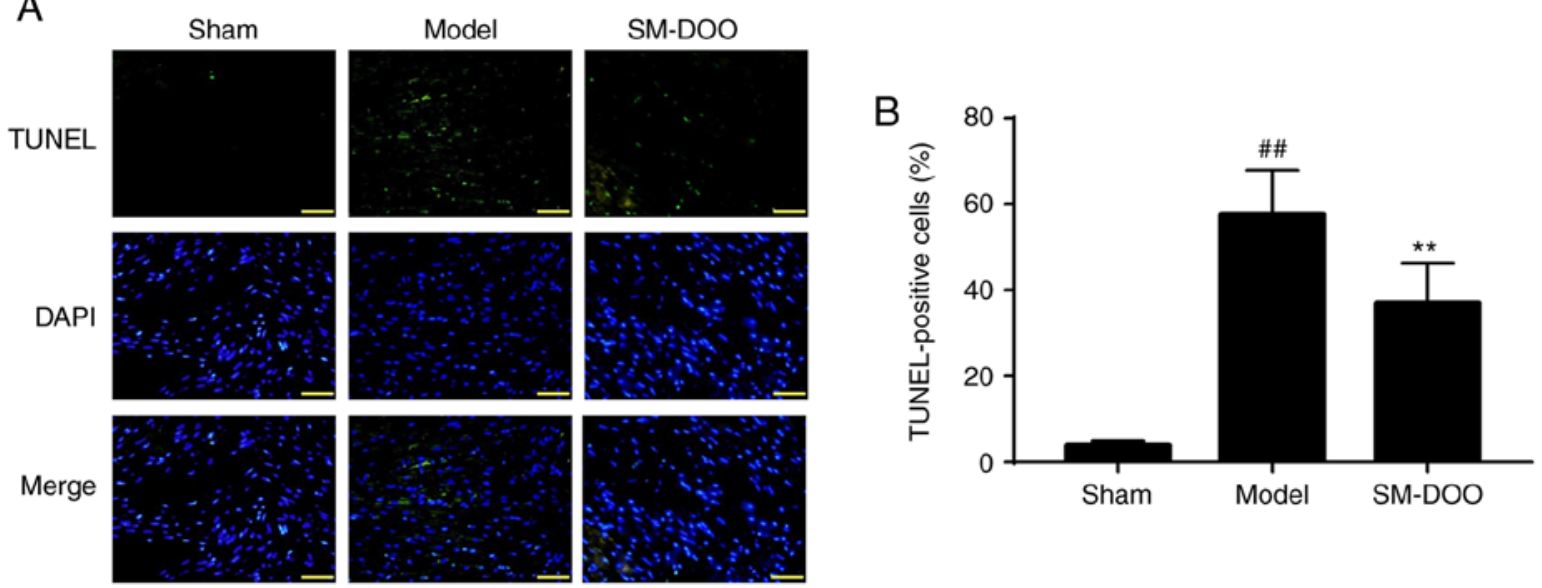

C

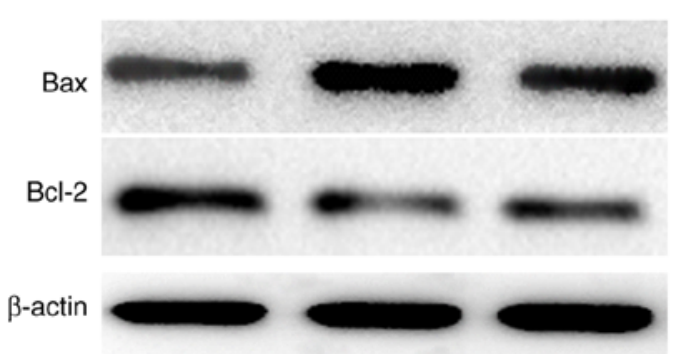

D

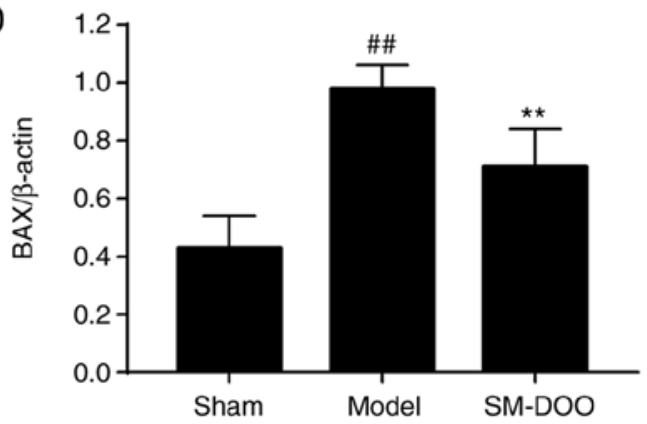

E

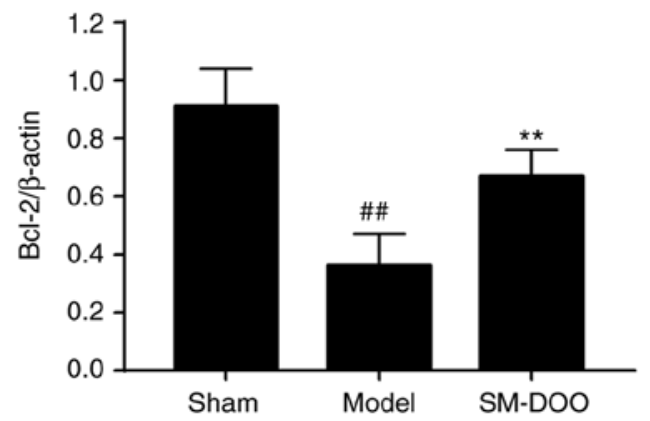

$\mathrm{F}$

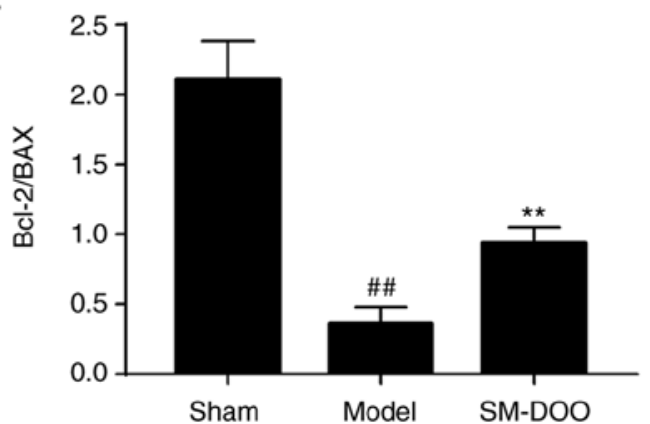

Figure 8. Effects of SM-DOO on the number of apoptotic cells following chronic myocardial ischemia. (A) Representative images of TUNEL staining in the sham, model and SM-DOO groups (magnification, $\mathrm{x} 400$ ). Apoptotic cells were detected by TUNEL staining (green). DAPI staining (blue) indicates the total number of nuclei. (B) The percentage of TUNEL-positive cell were expressed as (apoptotic cells/total nuclei) x 100. (C) Representative western blots of Bax and Bcl-2. Relative density of (D) Bax and (E) Bcl-2 normalized to $\beta$-actin. (F) Analysis of the Bcl-2/Bax ratio. All values are presented as the mean \pm standard deviation. ${ }^{\# \#} \mathrm{P}<0.01$ vs. Sham group; ${ }^{* *} \mathrm{P}<0.01$ vs. Model group. SM, Radix Salvia miltiorrhiza; DOO, Lignum Dalbergia odorifera volatile oil; TUNEL, terminal deoxynucleotidyl transferase-mediated dUTP nick end-labeling; Bax, Bcl-2-associated X.

Fig. 8B $(\mathrm{P}<0.01)$. To further examine the potential mechanisms of the anti-apoptotic effect of SM-DOO, the expression of Bax and Bcl-2 was determined. As shown in Fig. 8C, D and E, the expression of Bax was significantly increased and $\mathrm{Bcl}-2$ was significantly decreased in the Model group. In addition, the Bcl-2/Bax ratio was significantly decreased in the Model group (Fig. 8F; P $<0.01$ ). Thus, treatment with SM-DOO ameliorated the effects of chronic myocardial ischemia on apoptosis.

Effect of SM-DOO on Akt and GSK-3 $\beta$ activation. To investigate the signaling pathways involved in the cardioprotective effects of SM-DOO, the expression of Akt, p-Akt, GSK-3 $\beta$ and p-GSK-3 $\beta$ was determined. As shown in Fig. 9, compared with the Sham group, the expression of p-Akt and p-GSK-3 $\beta$ was slightly increased, while Akt and GSK-3 $\beta$ levels were not altered in the Model group. Compared with the Model group, the SM-DOO group had significantly higher levels of p-Akt/Akt and p-GSK-3 $\beta /$ GSK-3 $\beta(P<0.01)$, indicating that SM-DOO administration markedly increased p-Akt and p-GSK-3 $\beta$ levels.

Detection of SM and DOO components by chromatography. By comparing the retention times of mixed standard solutions with SM (Fig. 10A), 8 components were identified as danshensu, rosmarinic acid, salvianolic acid B, salvianolic acid A, tanshinone $\mathrm{II}_{\mathrm{A}}$ silate sodium, dihydrotanshinone I, cryptotanshinone and tanshinone $\mathrm{II}_{\mathrm{A}}$ by chromatography (Fig. 10B). The main components of SM were salvianolic acid B (18.67\%), cryptotanshinone $(11.83 \%)$ and tanshinone $\mathrm{II}_{\mathrm{A}}(10.55 \%)$. A total of 
A

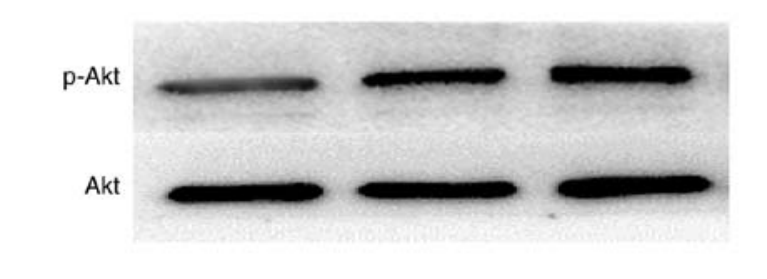

C

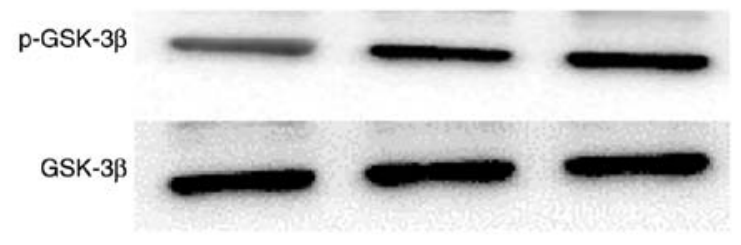

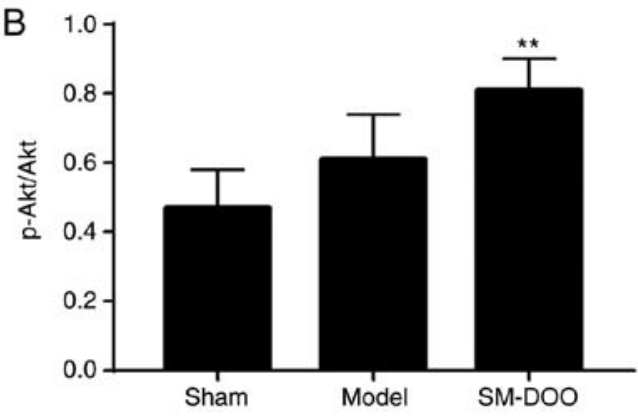

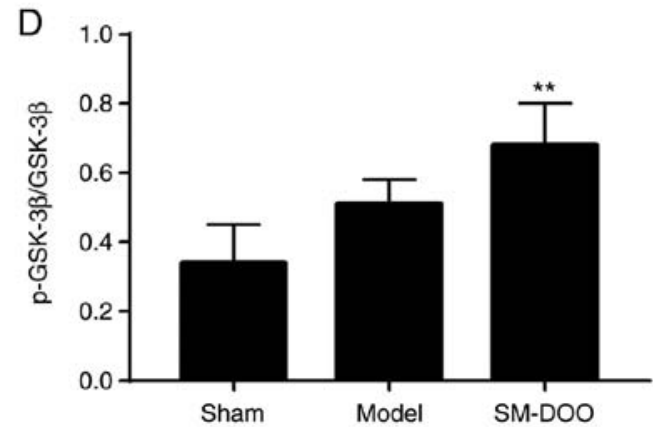

Figure 9. Akt, p-Akt, GSK-3 $\beta$ and p-GSK-3 $\beta$ protein levels in the myocardial tissue of the Sham, Model and SM-DOO groups were measured using western blotting. (A) Representative western blotting results for Akt and p-Akt. (B) Analysis of the p-Akt/Akt ratio. (C) Representative western blotting results for GSK-3 $\beta$ and p-GSK-3 $\beta$. (D) Analysis of the p-GSK-3 $\beta /$ GSK-3 $\beta$ ratio. All values are presented as the mean \pm standard deviation. ${ }^{* *} \mathrm{P}<0.01$ vs. Model group. p-, phosphorylated-; GSK, glycogen synthase kinase; SM, Radix Salvia miltiorrhiza; DOO, Lignum Dalbergia odorifera volatile oil.

Table II. Components of Lignum Dalbergia odorifera volatile oil as determined by gas chromatography coupled with mass spectrometry.

\begin{tabular}{llccc}
\hline No. & Compound name & Fomula & Retention time (min) & Relative mass fraction (\%) \\
\hline 1 & Pinene & $\mathrm{C}_{10} \mathrm{H}_{16}$ & 6.216 & 1.89 \\
2 & Terpinolene & $\mathrm{C}_{10} \mathrm{H}_{16}$ & 10.841 & 4.71 \\
3 & Eucalyptol & $\mathrm{C}_{10} \mathrm{H}_{18} \mathrm{O}$ & 12.216 & 2.38 \\
4 & Eugenol & $\mathrm{C}_{10} \mathrm{H}_{12} \mathrm{O}_{2}$ & 31.883 & 3.49 \\
5 & $\beta$-Farnesene & $\mathrm{C}_{15} \mathrm{H}_{24}$ & 42.945 & 5.16 \\
6 & $\alpha$-santalol & $\mathrm{C}_{15} \mathrm{H}_{24} \mathrm{O}$ & 56.260 & 9.01 \\
7 & $\alpha$-Farnesene & $\mathrm{C}_{15} \mathrm{H}_{24}$ & 57.368 & 2.63 \\
8 & Nerolidol & $\mathrm{C}_{15} \mathrm{H}_{26} \mathrm{O}$ & 61.348 & 22.52 \\
9 & Caryophyllene oxide & $\mathrm{C}_{15} \mathrm{H}_{24} \mathrm{O}$ & 62.001 & 12.70 \\
\hline
\end{tabular}

9 chemical components of DOO were detected by GC-MS and identified by NIST Database retrieval, as shown in Table II. The major components of DOO were nerolidol $(22.52 \%)$, caryophyllene oxide $(12.70 \%), \alpha$-santalol $(9.01 \%)$ and $\beta$-farnesene (5.16\%). The total ion current (TIC) of DOO is given in Fig. 10C.

\section{Discussion}

Myocardial ischemia has become one of the most common cardiovascular diseases, and it is difficult to treat in the clinic. The beneficial effects of SM-DOO in the prevention and treatment of heart disease have been demonstrated. However, little has been reported regarding the potential cardioprotective effects of SM-DOO in chronic myocardial ischemia. To address this problem, a clinically relevant animal model was used in the present study to evaluate the cardioprotective effects of SM-DOO in chronic myocardial ischemia.

Small animal models have been extensively used in cardiovascular research (19-21). However, they bear only a partial resemblance to humans, which may not translate to clinical trials (22). Pig models are widely used to research cardiovascular disease because of the similarities between pig and human hearts, such as cardiac anatomy and function, hemodynamics, coronary circulation, drug dosing, pharmacokinetics and a lack of preexisting collateral circulation (23). An ameroid constrictor is a stainless steel jacket with a casein bar in its central lumen. Dehydrated casein has a tendency to absorb fluid and swell, thus gradually leading to the chronic myocardial ischemia by obstructing the coronary 

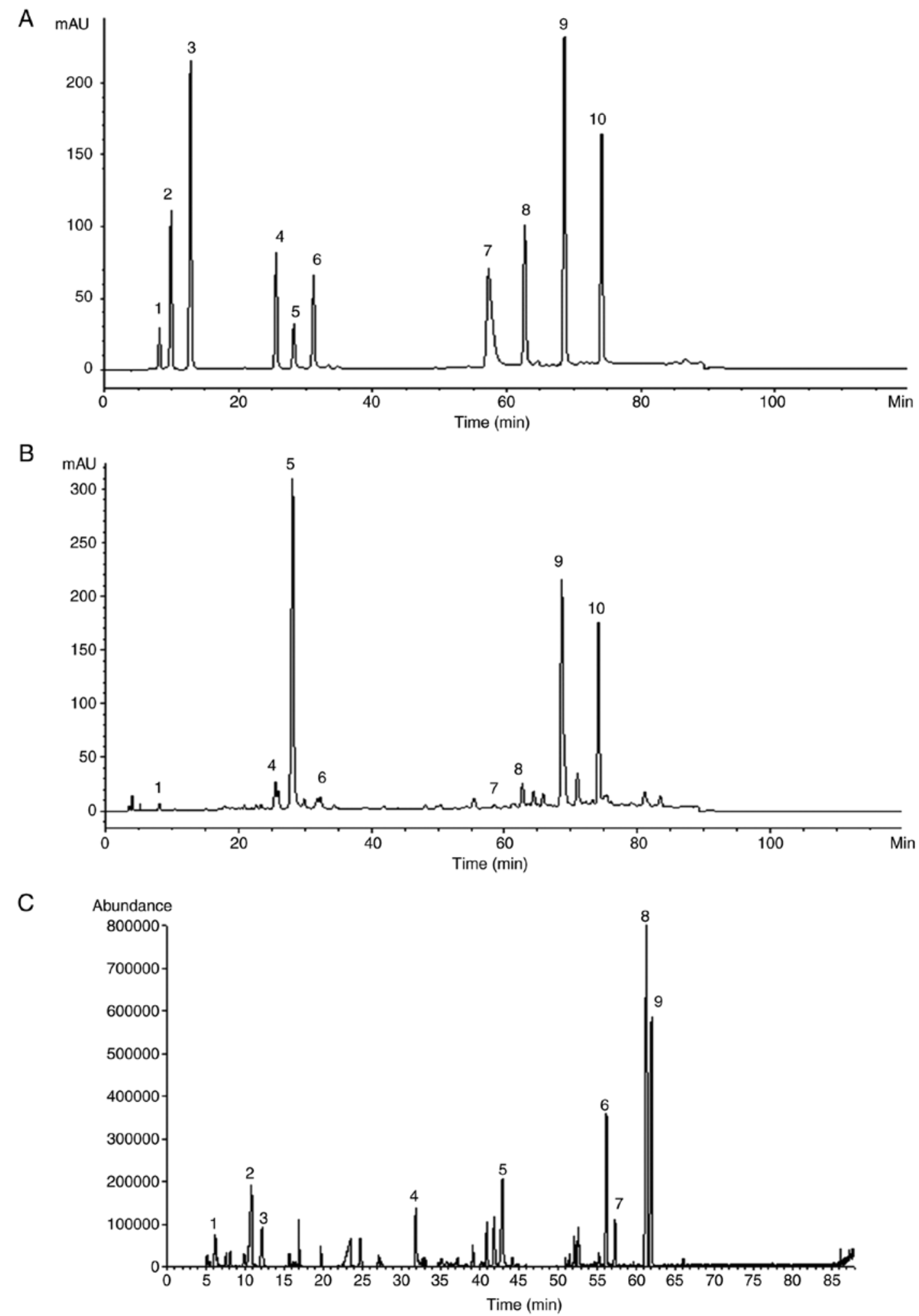

Figure 10. Representative chromatograms of the identified components in (A) mixed standard solutions of SM and (B) the SM extract, including 1. danshensu, 2. protocatechuic acid, 3. protocatechuic aldehyde, 4. rosmarinic acid, 5. salvianolic acid B, 6. salvianolic acid A, 7. tanshinone IIA silate sodium, 8. dihydrotanshinone $\mathrm{I}, 9$. cryptotanshinone and 10 . tanshinone $\mathrm{II}_{\mathrm{A}}$. (C) Total ion chromatogram of DOO, as determined by gas chromatography coupled with mass spectrometry. SM, Radix Salvia miltiorrhiza; DOO, Lignum Dalbergia odorifera volatile oil.

artery (24-27). In the present study, an X-ray coronary angiography demonstrated that the chronic myocardial ischemia model was stably established.

CK-MB, LDH, Myo and cTnI levels are considered to be important indicators of myocardial damage, and are signifi- cantly increased by blockage of the LAD (28-32). In addition, AST and ALT have previously been used to diagnose coronary heart disease $(33,34)$. The elimination and release of myocardial injury markers occurred at different times, suggesting that the combined analysis of myocardial injury markers may 
provide more precise information to influence therapeutic decisions (35). Thus, sensitive myocardial injury markers, including CK-MB, LDH, ALT, AST, cTnI and Myo, were measured to determine whether SM-DOO alleviated the degree of myocardial ischemia. As expected, SM-DOO significantly reduced changes in these markers, demonstrating an amelioration of myocardial injury. Furthermore, the echocardiographic (LVEDd, LVEDs, EDV, ESV, EF and FS) and hemodynamic (MAP, HR, LVSP, LVEDP, $+\mathrm{dp} / \mathrm{dt}_{\max }$ and $-\mathrm{dp} / \mathrm{dt}_{\max }$ ) parameters suggested that SM-DOO had a beneficial effect on preventing the impairment of cardiac function. In addition, LAD occlusion leads to myocardial injury and metabolic acidosis, decreasing the levels of $\mathrm{PO}_{2}$ and increasing $\mathrm{PCO}_{2}(29,36)$. In the present study, SM-DOO significantly prevented these changes, ameliorating myocardial oxygen consumption. Severe myocardial ischemia is associated with microstructural abnormalities in the myocardium (37). Pre-treatment with SM-DOO decreased the infarct size and reduced the extent of myocardial damage, supporting that conclusion that SM-DOO provided cardioprotective effects.

The results of the present study have determined that SM-DOO has a cardioprotective effect against chronic myocardial ischemia. Next, the potential mechanisms of these effects were explored. Apoptosis plays an important role in cardiac injury and has become a focus of research (38). The expression of several apoptosis-related genes is altered in ischemic tissue, including $\mathrm{Bax}$ and $\mathrm{Bcl}-2$, which play important roles in apoptosis (39). The Bcl-2 family members are key regulators of physiological and pathological apoptosis, including cell death promoters such as Bax and Bcl-2 associated agonist of cell death, and cell death inhibitors such as Bcl-2, Bcl-X and Mcl-1 (40). Changes in the Bcl-2/Bax ratio leads to the induction of apoptotic cell death (41). In the present study, the anti-apoptotic effects of SM-DOO may be mediated by inhibiting the upregulation of Bax and downregulation of Bcl-2. This finding is consistent with the results of the TUNEL assay. These results suggest that SM-DOO may exert its cardioprotective effect against myocardial injury following chronic myocardial ischemia by preventing apoptosis.

The PI3K/Akt pathway is particularly important in mediating myocardial cell survival. Studies have shown that activation of the PI3K/Akt signaling pathway is essential for anti-apoptotic and cardioprotective effects $(42,43)$. GSK-3 is a serine/threonine kinase with versatile cellular functions in the heart, including in gene expression, hypertrophy and apoptosis (44). GSK-3 $\beta$ is active in cardiomyocytes unstimulated by ischemia and ischemia/reperfusion, and sensitizes cells to death-promoting insults, which may be an underlying mechanism of cardioprotection (45). Akt phosphorylates and subsequently inactivates GSK-3 $\beta$, which contributes to the attenuation of myocardial injury (46). To investigate the involvement of the PI3K/Akt signaling pathway on the cardioprotective effects of SM-DOO treatment, the expression of Akt and GSK-3 $\beta$ was determined using western blotting. As shown in the results, pretreatment with SM-DOO significantly increased the relative p-Akt and p-GSK-3 $\beta$ levels compared with the Model group. These findings suggest that the PI3K/Akt/GSK-3 $\beta$ signaling pathway may be involved in the cardioprotective effects of SM-DOO in a chronic myocardial ischemia model. However, further studies utilizing an inhibitor of PI3K/Akt are required to validate the involvement of this pathway.

The chemical composition of SM includes water-soluble and lipid-soluble components $(47,48)$. Using HPLC analysis, 8 components of SM were identified by comparing its retention time with the standards. The main components of SM were salvianolic acid B, cryptotanshinone and tanshinone $\mathrm{II}_{\mathrm{A}}$. DOO was also analyzed using the established GC-MS method. The majority of the components, including pinene, terpinolene, eucalyptol, eugenol, $\beta$-farnesene, $\alpha$-santalol, $\alpha$-farnesene, nerolidol and caryophyllene oxide, were identified by performing a similarity match with the NIST Database. Studies have demonstrated that various chemical components of SM, such as danshensu, salvianolic acid B, salvianolic acid A, rosmarinic acid and tanshinone IIA, have anti-apoptotic activities (49-52). In addition, recent research indicates that tanshinone IIA, dihydrotanshinone I, danshensu, salvianolic acid B and salvianolic acid A play a protective role by regulating GSK-3 $\beta$ (53-56). However, there are few studies regarding whether the chemical components of DOO achieve a protective effect by regulating GSK-3 $\beta$. We plan to explore the effects of active chemical constituents of SM-DOO against chronic myocardial ischemia in the future.

In conclusion, pretreatment with SM-DOO may ameliorate cardiac injury resulting from chronic myocardial ischemia. Myocardial injury markers (CK-MB, LDH, ALT, AST, cTnI and Myo), markers of myocardial oxygen consumption ( $\mathrm{pH}$, $\mathrm{PO}_{2}$ and $\mathrm{PCO}_{2}$ ), metrics of left ventricular function (LVEDd, LVEDs, EDV, ESV, EF, FS, MAP, HR, LVSP, LVEDP, $+\mathrm{dp} / \mathrm{dt}_{\max }$ and $\left.-\mathrm{dp} / \mathrm{dt}_{\max }\right)$ and indicators of myocardial tissue damage (TTC, H\&E, TUNEL and western blotting) revealed the effects of SM-DOO against chronic myocardial ischemia. Furthermore, the cardioprotective effects of SM-DOO may be partly mediated via activation of the PI3K/Akt/GSK-3 $\beta$ signaling pathway. The results of the present study may help to improve our understanding of the molecular mechanisms underlying the cardioprotective effects of SM-DOO, and provide a basis for the use of SM-DOO as an effective drug for the prophylaxis and treatment of chronic myocardial ischemia.

\section{Acknowledgements}

The authors would like to thank Beijing Key Laboratory of Pre-clinical Research and Evaluation for Cardiovascular Implant Materials (Fuwai Hospital, Chinese Academy of Medical Sciences) for providing the experimental animals and platform.

\section{Funding}

The present study was supported by the National Natural Science Foundation of China (grant nos. 81470174 and 31771265).

\section{Availability of data and materials}

All data generated or analyzed during this study are included in this published article. 


\section{Authors' contributions}

The present study was performed with collaboration between all authors. MX, YY and AW defined the research theme and revised the manuscript critically. RL, JD and FM designed the methods and experiments, performed the laboratory experiments and wrote the paper. HB, MNZ, MZ and YL collected and analyzed the data, and interpreted the results. All authors have read and approved the final manuscript.

\section{Ethics approval and consent to participate}

The Animal Welfare and Ethics Committee of Fuwai Hospital, Chinese Academy of Medical Sciences approved all experiments [approval no. 0072-1-27-HX (F)].

\section{Competing interests}

The authors declare that they have no competing interests.

\section{References}

1. Shimokawa $\mathrm{H}$ and Yasuda S: Myocardial ischemia: Current concepts and future perspectives. J Cardiol 52: 67-78, 2008.

2. Lv Y, Liu X, Yan S, Liang X, Yang Y, Dai W and Zhang W: Metabolomic study of myocardial ischemia and intervention effects of Compound Danshen Tablets in rats using ultra-performance liquid chromatography/quadrupole time-of-flight mass spectrometry. J Pharm Biomed Anal 52: $129-135,2010$.

3. Xu T, Wu X, Chen Q, Zhu S, Liu Y, Pan D, Chen X and Li D: The anti-apoptotic and cardioprotective effects of salvianolic acid a on rat cardiomyocytes following ischemia/reperfusion by DUSP-mediated regulation of the ERK1/2/JNK pathway. PLoS One 9: e102292, 2014.

4. JianXin C, Xue X, ZhongFeng L, Kuo G, FeiLong Z, ZhiHong L, Xian W and HongCai S: Qishen Yiqi Drop Pill improves cardiac function after myocardial ischemia. Sci Rep 6: 24383, 2016.

5. Zhang Y, Shi P, Yao H, Shao Q and Fan X: Metabolite profiling and pharmacokinetics of herbal compounds following oral administration of a cardiovascular multi-herb medicine (Qishen yiqi pills) in rats. Curr Drug Metab 13: 510-523, 2012.

6. Wu SD, Wang J, Chen SC, Xu JB, Zheng Q, Yan YF, Wen TM and Tang YR: Effect of Xiangdan Injection on mRNA expression of endothelial vaso-active factors of pa tients with coronary heart disease and blood stasis. Zhong Xi Yi Jie He Xue Bao 2: 94-96, 2004 (In Chinese).

7. Wang SX, Luo K, Liang J, Fan F, Li H, Zheng JB and Zheng XH: Metabolomics study on the synergistic interaction between Salvia miltiorrhiza and Lignum dalbergiae odoriferae used as 'Jun-Shi' herbs in a S. miltiorrhiza recipe. Med Chem Res 20: 16-22, 2011.

8. Sugiyama A, Zhu BM, Takahara A, Satoh Y and Hashimoto K: Cardiac effects of salvia miltiorrhiza/dalbergia odorifera mixture, an intravenously applicable Chinese medicine widely used for patients with ischemic heart disease in China. Circ J 66: 182-184, 2002.

9. Mu F, Duan J, Bian H, Zhai X, Shang P, Lin R, Zhao M, Hu D, Yin Y, Wen A, et al: Metabonomic strategy for the evaluation of Chinese medicine salvia miltiorrhiza and dalbergia odorifera interfering with myocardial ischemia/reperfusion injury in rats. Rejuvenation Res 20: 263-277, 2017.

10. Zheng X, Zhao X, Wang S, Luo K, Wei Y and Zheng J: Co-administration of Dalbergia odorifera increased bioavailability of Salvia miltiorrhizae in rabbits. Am J Chin Med 35: 831-840, 2007.

11. Zhou L, Zuo Z and Chow MS: Danshen: An overview of its chemistry, pharmacology, pharmacokinetics, and clinical use. J Clin Pharmacol 45: 1345-1359, 2005.

12. Liu R, Ye M, Guo H, Bi K and Guo DA: Liquid chromatography/electrospray ionization mass spectrometry for the characterization of twenty-three flavonoids in the extract of Dalbergia odorifera. Rapid Commun Mass Spectrom 19: $1557-1565,2005$.
13. Tu J and Song K: Curative effect observation on Xiangdan injection in the treatment of coronary artery disease. Chin Med Pharm 22: 110-111, 2011 (In Chinese).

14. Qin F and Huang X: Guanxin II for the management of coronary heart disease. Chin J Integr Med 15: 472-476, 2009.

15. Mu F, Duan J, Bian H, Yin Y, Zhu Y, Wei G, Guan Y, Wang Y, Guo C, Wen A, et al: Cardioprotective effects and mechanism of Radix Salviae miltiorrhizae and Lignum Dalbergiae odoriferae on rat myocardial ischemia/reperfusion injury. Mol Med Rep 16: 1759-1770, 2017.

16. Krishnamoorthy K and Subramaniam P: Phytochemical profiling of leaf, stem, and tuber parts of Solena amplexicaulis (Lam.) Gandhi using GC-MS. Int Sch Res Notices 2014: 567409, 2014.

17. Huang JH, Huang XZ, Chen ZY, Zheng QS and Sun RY: Dose conversion among different animals and healthy volunteers in pharmacological study. Chin J Clin Pharmacol Therap 9: 1069-1072, 2004.

18. Henriques JP, Zijlstra F, van 't Hof AW, de Boer MJ, Dambrink JH, Gosselink M, Hoorntje JC and Suryapranata H: Angiographic assessment of reperfusion in acute myocardial infarction by myocardial blush grade. Circulation 107: 2115-2119, 2003.

19. Liu Z, Chen JM, Huang H, Kuznicki M, Zheng S, Sun W, Quan N, Wang L, Yang H, Guo HM, et al: The protective effect of trimetazidine on myocardial ischemia/reperfusion injury through activating AMPK and ERK signaling pathway. Metabolism 65: 122-130, 2016.

20. Saito N: Letter by Saito regarding article, 'Collateral donor artery physiology and the influence of a chronic total occlusion on fractional flow reserve'. Circ Cardiovasc Interv 8: e002794, 2015.

21. Zhao HW, Qin F, Liu YX, Huang X and Ren P: Antiapoptotic mechanisms of Chinese medicine formula, Guan-Xin-Er-Hao, in the rat ischemic heart. Tohoku J Exp Med 216: 309-316, 2008.

22. Seok J, Warren HS, Cuenca AG, Mindrinos MN, Baker HV, Xu W, Richards DR, McDonald-Smith GP, Gao H, Hennessy L, et al: Genomic responses in mouse models poorly mimic human inflammatory diseases. Proc Natl Acad Sci USA 110: 3507-3512, 2013.

23. Elmadhun NY, Sabe AA, Robich MP, Chu LM, Lassaletta AD and Sellke FW: The pig as a valuable model for testing the effect of resveratrol to prevent cardiovascular disease. Ann NY Acad Sci 1290: 130-135, 2013.

24. Ikonen TS, Pätilä T, Virtanen K, Lommi J, Lappalainen K, Kankuri E, Krogerus L and Harjula A: Ligation of ameroid-stenosed coronary artery leads to reproducible myocardial infarction-a pilot study in a porcine model. J Surg Res 142: 195-201, 2007.

25. Caillaud D, Calderon J, Réant P, Lafitte S, Dos Santos P, Couffinhal T, Roques X and Barandon L: Echocardiographic analysis with a two-dimensional strain of chronic myocardial ischemia induced with ameroid constrictor in the pig. Interact Cardiovasc Thorac Surg 10: 689-693, 2010.

26. Tuzun E, Oliveira E, Narin C, Khalil H, Jimenez-Quevedo P, Perin E and Silva G: Correlation of ischemic area and coronary flow with ameroid size in a porcine model. J Surg Res 164: 38-42, 2010.

27. von Degenfeld G, Raake P, Kupatt C, Lebherz C, Hinkel R, Gildehaus FJ, Münzing W, Kranz A, Waltenberger J, Simoes M, et al: Selective pressure-regulated retroinfusion of fibroblast growth factor- 2 into the coronary vein enhances regional myocardial blood flow and function in pigs with chronic myocardial ischemia. J Am Coll Cardiol 42: 1120-1128, 2003.

28. Eisenman A: Troponin assays for the diagnosis of myocardial infarction and acute coronary syndrome: Where do we stand. Expert Rev Cardiovasc Ther 4: 509-514, 2006.

29. Li X, Shao D, Wang G, Jiang T, Wu H, Gu B, Cao K, Zhang J, Qi L and Chen Y: Effects of different LAD-blocked sites on the development of acute myocardial infarction and malignant arrhythmia in a swine model. J Thorac Dis 6: 1271-1277, 2014.

30. Tsaroucha A, Chondrogiannis C, Mani A and Staikou C: Myocardial involvement during ischemia-induced acute liver failure in the pig. J Invest Surg 26: 99-104, 2013.

31. Arya DS, Bansal P, Ojha SK, Nandave M, Mohanty I and Gupta SK: Pyruvate provides cardioprotection in the experimental model of myocardial ischemic reperfusion injury. Life Sci 79: 38-44, 2006.

32. Doganci S, Yildirim V, Bolcal C, Korkusuz P, Gumusel B, Demirkilic U and Aydin A: Sodium nitrite and cardioprotective effect in pig regional myocardial ischemia-reperfusion injury model. Adv Clin Exp Med 21: 713-726, 2012. 
33. Elizondo-Montemayor L, Ugalde-Casas PA, Lam-Franco L Bustamante-Careaga H, Serrano-González M, Gutiérrez NG and Martínez U: Association of ALT and the metabolic syndrome among Mexican children. Obes Res Clin Pract 8: e79-e87, 2014.

34. Xu Q, Higgins T and Cembrowski GS: Limiting the testing of AST: A diagnostically nonspecific enzyme. Am J Clin Pathol 144: 423-426, 2015.

35. Solymoss BC, Bourassa MG, Fortier A and Théroux P: Evaluation and risk stratification of acute coronary syndromes using a low cut-off level of cardiac troponin T, combined with CK-MB mass determination. Clin Biochem 37: 286-292, 2004.

36. Zhang M, DongW, Lu S ,Yu Y, Liu H, Li G, Qiang W, Wang L and Lou J: Study on blood-gas changes in the coronary artery of myocardial ischemia reperfusion injury of rabbit. J Naval Med College 1: 9-12, 1996 (In Chinese).

37. Feng YJ, Chen C, Fallon JT, Lai T, Chen L, Knibbs DR, Waters DD and Wu AH: Comparison of cardiac troponin I, creatine kinase-MB and myoglobin for detection of acute ischemic myocardial injury in a swine model. Am J Clin Pathol 110: 70-77, 1998

38. Ishihara Y and Shimamoto N: Sulfaphenazole attenuates myocardial cell apoptosis accompanied with cardiac ischemia-reperfusion by suppressing the expression of BimEL and Noxa. J Pharmacol Sci 119: 251-259, 2012

39. Qu D, Han J, Ren H, Yang W, Zhang X, Zheng Q and Wang D Cardioprotective effects of Astragalin against myocardial ischemia/reperfusion injury in isolated rat heart. Oxid Med Cell Longev 2016: 8194690, 2016.

40. Pan H, Li D, Fang F, Chen D, Qi L, Zhang R, Xu T and Sun H: Salvianolic acid A demonstrates cardioprotective effects in rat hearts and cardiomyocytes after ischemia/reperfusion injury. J Cardiovasc Pharmacolm 58: 535-542, 2011.

41. Zhang Q, Wang G, Yuan W, Wu J, Wang M and Li C: The effects of phosphodiesterase-5 inhibitor sildenafil against post-resuscitation myocardial and intestinal microcirculatory dysfunction by attenuating apoptosis and regulating microRNAs expression: Essential role of nitric oxide syntheses signaling. J Transl Med 13: 177, 2015.

42. Wang ZG, Wang Y, Huang Y, Lu Q, Zheng L, Hu D, Feng WK, Liu YL, Ji KT, Zhang HY, et al: bFGF regulates autophagy and ubiquitinated protein accumulation induced by myocardial ischemia/reperfusion via the activation of the PI3K/Akt/mTOR pathway. Sci Rep 5: 9287, 2015.

43. Liu S, Ai Q, Feng K, Li YB and Liu X: The cardioprotective effect of dihydromyricetin prevents ischemia-reperfusion-induced apoptosis in vivo and in vitro via the PI3K/Akt and HIF-1 $\alpha$ signaling pathways. Apoptosis 21: 1366-1385, 2016.

44. Zhai P, Sciarretta S, Galeotti J, Volpe M and Sadoshima J: Differential roles of GSK-3 $\beta$ during myocardial ischemia and ischemia_reperfusion. Circ Res 109: 502-511, 2011.

45. Duan J, Guan Y, Mu F, Guo C, Zhang E, Yin Y, Wei G, Zhu Y, Cui J, Cao J, et al: Protective effect of butin against ischemia/reperfusion-induced myocardial injury in diabetic mice: Involvement of the AMPK/GSK-3 $\beta / \mathrm{Nrf} 2$ signaling pathway. Sci Rep 7: 41491, 2017.
46. Nagaoka K, Matoba T, Mao Y, Nakano Y, Ikeda G, Egusa S, Tokutome M, Nagahama R, Nakano K, Sunagawa K, et al: A new therapeutic modality for acute myocardial infarction: Nanoparticle-mediated delivery of Pitavastatin induces cardioprotection from ischemia-reperfusion injury via activation of PI3K/Akt pathway and anti-inflammation in a rat model. PLoS One 10: e0132451, 2015.

47. Liu ZJ, Shi ZL, Tu C, Zhang HZ, Gao D, Li CY, He Q, Li RS, Guo YM, Niu M, et al: An activity-calibrated chemical standardization approach for quality evaluation of Salvia miltiorrhiza Bge. RSC Adv 7: 5331-5339, 2017.

48. Zhou GJ, Wang W, Xie XM, Qin MJ, Kuai BK and Zhou TS: Post-harvest induced production of salvianolic acids and significant promotion of antioxidant properties in roots of Salvia miltiorrhiza (Danshen). Molecules 19: 7207-7222, 2014.

49. Fan G, Yu J, Asare PF, Wang L, Zhang H, Zhang B, Zhu Y and Gao X: Danshensu alleviates cardiac ischaemia/reperfusion injury by inhibiting autophagy and apoptosis via activation of mTOR signalling. J Cell Mol Med 20: 1908-1919, 2016.

50. Jia LQ, Yang GL, Ren L, Chen WN, Feng JY, Cao Y, Zhang L, Li XT and Lei P. Tanshinone IIA reduces apoptosis induced by hydrogen peroxide in the human endothelium-derived EA.hy 926 cells. J Ethnopharmacol 143: 100-108, 2012.

51. Zhuang P, Zhang Y, Cui G, Bian Y, Zhang M, Zhang J, Liu Y, Yang X, Isaiah AO, Lin Y, et al: Direct stimulation of adult neural stem/progenitor cells in vitro and neurogenesis in vivo by salvianolic acid B. PLoS One 7: e35636, 2012.

52. Li XL, Liu JX, Li P and Zheng YQ: Protective effect of rosmarinic acid on hypoxia/reoxygenation injury in cardiomyocytes. Zhongguo Zhong Yao Za Zhi 39: 1897-1901, 2014 (In Chinese).

53. Jung SH, Seol HJ, Jeon SJ, Son KH and Lee JR: Insulin-sensitizing activities of tanshinones, diterpene compounds of the root of Salvia miltiorrhiza Bunge. Phytomedicine 16: 327-335, 2009.

54. Guo C, Yin Y, Duan J, Zhu Y, Yan J, Wei G, Guan Y, Wu X, Wang Y, Xi M and Wen A: Neuroprotective effect and underlying mechanism of sodium danshensu [3-(3,4-dihydroxyphenyl) lactic acid from Radix and Rhizoma Salviae miltiorrhizae = Danshen] against cerebral ischemia and reperfusion injury in rats. Phytomedicine 22: 283-289, 2015.

55. Gao Q, Hu X, Jiang XJ, Guo MJ, Ji H, Wang Y and Fan YC: Cardiomyocyte-like cells differentiation from non $\beta$-catenin expression mesenchymal stem cells. Cytotechnology 66: 575-584, 2014

56. Li XL, Fan JP, Liu JX and Liang LN: Salvianolic acid A protects neonatal cardiomyocytes against hypoxia/reoxygenation-induced injury by preserving mitochondrial function and activating Akt/GSK-3beta signals. Chin J Integr Med: 1-8, 2017.

This work is licensed under a Creative Commons Attribution-NonCommercial-NoDerivatives 4.0 International (CC BY-NC-ND 4.0) License. 